\title{
Inference and Learning in Planning
}

\author{
Hector Geffner \\ ICREA \& Universitat Pompeu Fabra \\ C/Roc Boronat 138, E-08018 Barcelona, Spain \\ hector .geffner@upf .edu \\ http://www.tecn.upf.es/ ${ }^{\sim}$ geffner
}

\begin{abstract}
Planning is concerned with the development of solvers for a wide range of models where actions must be selected for achieving goals. In these models, actions may be deterministic or not, and full or partial sensing may be available. In the last few years, significant progress has been made, resulting in algorithms that can produce plans effectively in a variety of settings. These developments have to do with the formulation and use of general inference techniques and transformations. In this invited talk, I'll review the inference techniques used for solving individual planning instances from scratch, and discuss the use of learning methods and transformations for obtaining more general solutions.
\end{abstract}

